\title{
A New Compact Soft X-ray Spectrometer for Resonant Inelastic X-ray Scattering Studies at PETRA III
}

\author{
Z. Yin ${ }^{1,2, a)}$, H. B. Peters ${ }^{3}$, U. Hahn ${ }^{1}$, M. Agåker ${ }^{4}$, A. Hage ${ }^{1,5}$, R. Reininger ${ }^{6}$, F. Siewert ${ }^{7}$, \\ J. Nordgren ${ }^{4}$, J. Viefhaus ${ }^{1}$ and S. Techert ${ }^{1,2,8, b)}$ \\ ${ }^{1}$ Photon Science, Deutsches Elektronen-Synchrotron (DESY), 22607 Hamburg, Germany \\ ${ }^{2}$ Structural Dynamics of (Bio)chemical Systems, Max Planck Institute for Biophysical Chemistry, 37077 Göttingen, Germany \\ ${ }^{3}$ ZM1, Deutsches Elektronen-Synchrotron (DESY), 22607 Hamburg, Germany \\ ${ }_{5}^{4}$ Department of Physics, Uppsala University,75121 Uppsala, Sweden \\ ${ }^{5}$ School of Mathematics and Physics, Queen's University, Belfast BT7 1NN, United Kingdom \\ ${ }^{6}$ Advanced Photon Source, Argonne National Laboratory, Argonne, Illinois 60439, USA \\ ${ }^{7}$ Institute Nanometre Optics and Technology, Helmholtz Zentrum Berlin, 12489 Berlin, Germany \\ ${ }^{8}$ Institute of X-ray Physics, Georg-August University, 37077 Göttingen, Germany
}

(Received XXXXX; accepted XXXXX; published online XXXXX)

(Dates appearing here are provided by the Editorial Office)

\begin{abstract}
We present a newly designed compact grating spectrometer for the energy range from $210 \mathrm{eV}$ to $1250 \mathrm{eV}$, which would include the $\mathrm{K} \alpha_{1,2}$ emission lines of vital elements like $\mathrm{C}, \mathrm{N}$ and $\mathrm{O}$. The spectrometer is based on a grazing incidence spherical varied line spacing (VLS) grating with $2400 \mathrm{l} / \mathrm{mm}$ at its center and a radius of curvature of $58542 \mathrm{~mm}$. First results show a resolving power of around 1000 at an energy of $550 \mathrm{eV}$ and a working spectrometer for high vacuum $\left(10^{-4} \mathrm{mbar}\right)$ environment without losing photon intensity.
\end{abstract}

\section{INTRODUCTION}

The rapid development of brilliant X-ray sources, like the third generation of synchrotron or free electron laser facilities, opens the door for new scientific techniques to elucidate the fundamental understanding of nature on the atomic level. One of the techniques is X-ray emission spectroscopy (XES) and a special case of XES is the socalled resonant inelastic scattering (RIXS). It has been utilized to project the valence orbital density of state on the core orbitals of molecules, showing the electronic structure of molecules and making it orbital, element and chemical environment sensitive, in addition to being site specific. ${ }^{1,2}$ Furthermore, RIXS is providing the possibility to clock nuclear and electronic wavepacket dynamics on the core hole lifetime, which, depending on the element, is in the range of a few femtoseconds. ${ }^{3,4}$ Therefore, XES and RIXS have been a popular method to study the electronic structure of chemical systems, $3 \mathrm{~d}$ transition metal etc. ${ }^{5-15}$ While XES and RIXS in the hard X-ray regime are detectable with crystal spectrometers using the Bragg law, 16,17 in the soft X-rays the needed lattice constant of crystals is too high and the quality of the planes of these crystals is not sufficient for high energy resolution. Early pioneering work by Callcott et al. ${ }^{18}$ and Nordgren et al. ${ }^{19}$ introduced a grating spectrometer based on the Rowland circle principle as the answer to perform high resolution XES and RIXS measurements in the soft X-ray regime, opening the possibility to study the $\mathrm{K}$ lines of systems containing vital elements like $\mathrm{C}, \mathrm{N}$ and $\mathrm{O}$ and $\mathrm{L}$ lines of $3 \mathrm{~d}$ metals. ${ }^{5-13}$ In recent years, new high resolution soft X-ray spectrometers based on varied line spacing gratings have been build. ${ }^{20-25}$ Some are even able to resolve vibrational states in the spectrum. ${ }^{26}$ Yet, they are huge setups with detector arm length of up to $5 \mathrm{~m} .^{22}$ Future spectrometer projects are even planned with detector arm length up to $15 \mathrm{~m}$ with the aim to reach higher resolving power.

The purpose of this paper is to present a new compact grazing incident flat field spectrometer using a spherical varied line spacing (SVLS) grating manufactured by HITACHI (001-0659) and delivering medium resolution. Compared to the widely established grating spectrometer based on Rowland geometry, the VLS grating minimizes aberrations and offers the advantage to focus on a flat focal plane which makes the utilization of X-ray CCDs possible. ${ }^{27}$ Optimization becomes much easier since the focus depends on the distance of the position-sensitive soft $\mathrm{X}$-ray detector and the grating. The height of the detector relative to the grating determines the energy. A detailed description of the theory how VLS grating works can be found here. ${ }^{22,28}$ As a 2D detector, an X-ray CCD from Andor with a chip size of $13.3 \times 13.3 \mathrm{~mm}^{2}$ and a pixel size of $13 \times 13 \mu \mathrm{m}^{2}$ has been used. ${ }^{29}$

The grating spectrometer described here combines a stable, flexible, and compact design with sufficient energy resolution for chemical and material science applications. Furthermore, the spectrometer can sustain large differential pressures making it compatible with liquid jet and gas applications. 


\section{THE VLS GRATING}

The advantage of a spherical VLS grating is the combination of dispersion and focusing in a single optical element. The groove spacing, $\sigma(\mathrm{w})$, as the function of the position $\mathrm{w}$ along the grating is given by ${ }^{30}$

$$
\sigma(w)=\frac{\sigma_{0}}{\left(1+\frac{2 b_{2} w}{R}+\frac{3 b_{3} w^{2}}{R^{2}}+\frac{4 b_{4} w^{3}}{R^{3}}\right)},(1)
$$

where $\sigma_{0}$ is the groove spacing at the center of the grating, $\mathrm{R}$ is the radius of curvature and $\mathrm{b}_{2}, \mathrm{~b}_{3}, \mathrm{~b}_{4}$, can be chosen to zero the defocus, coma, and spherical aberrations, respectively.

The parameters of the grating given by the manufacturer are $R=57680 \mathrm{~mm}, b_{2}=-95.97, b_{3}=9.492 \times 10^{3}$ and $b_{4}=-9.78 \times 10^{5}$. The effective ruled area of the gold coated grating is $60 \times 30 \mathrm{~mm}^{2}$.

The grating was inspected using an atomic force microscope (AFM), ${ }^{31,32}$ which is a scanning probe microscopy technique providing height resolution on an atomic scale. ${ }^{33}$ The AFM allows to characterize the relevant parameters of the gratings like groove profile, micro-roughness on the grooves and groove density, (see Figure 1). The imperfect shape of the grooves causes lower diffraction efficiency.
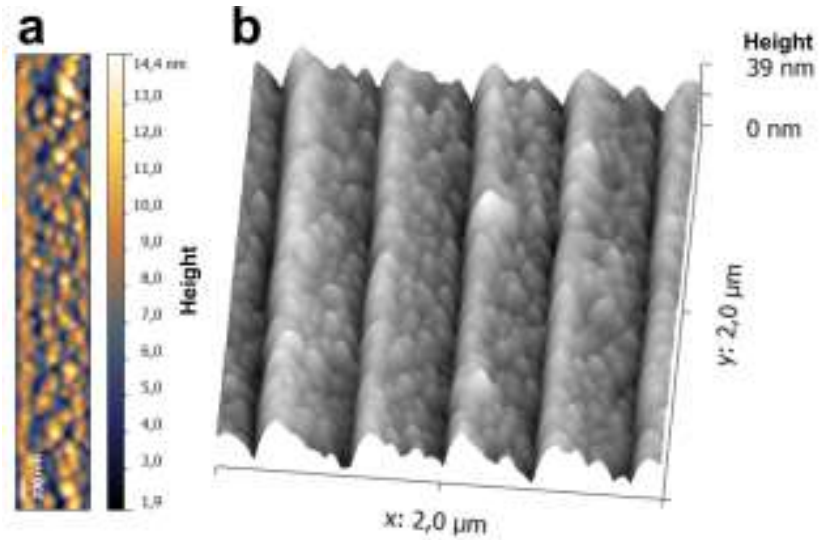

Figure 1: The left figure shows the topography on one selected groove in terms of height. The rms microroughness is $2.06 \mathrm{~nm}$ on this section. The right figure shows the topography and blaze profile of the grating which is typical for a replica grating etched into the grating blank and coated with Au.

The instrument used for these measurements is a Bruker SIS-Ultraobjective AFM with a $40 \mathrm{x} 40 \mu \mathrm{m}^{2}$ scanner. The instrument is based on a PICO-Station system with an active vibration damping.

The tip used in the measurements is a silicon SPM-sensor for non-contact mode, its resonance frequency is $190 \mathrm{kHz}$ and its force constant $48 \mathrm{~N} / \mathrm{m}$. The tip is shaped like a polygon-based pyramid with a height of $10-15 \mu \mathrm{m}$. The tip-radius is less than $8 \mathrm{~nm}$. Thus, the achievable lateral resolution is in the range of about $20 \mathrm{~nm} \mathrm{-} \mathrm{a}$ conservative estimate. The AFM tip was replaced after ten scans to avoid the results to be affected by the tip wear. The measured micro-roughness, approximately $2 \mathrm{~nm} \mathrm{rms}$, is a typical value for standard gratings manufactured by different vendors. ${ }^{34}$ The measure blaze angle is listed in table I.

The grating's residual slope error and its radius of curvature were measured with the BESSY-NOM slope measuring profiler. ${ }^{34}$ The slope error in the spatial frequency range between $1 \mathrm{~mm}^{-1}$ up to the aperture length cause aberrations in the optical system. The measured values are presented in table I.

\begin{tabular}{|c|c|c|c|c|c|c|}
\hline $\begin{array}{c}\sigma_{0} \\
{[\mathrm{~mm}]}\end{array}$ & $\begin{array}{c}\mathrm{R} \\
{[\mathrm{mm}]^{*}}\end{array}$ & $\begin{array}{l}\text { Blaze } \\
\text { Angle } \\
{\left[{ }^{\circ}\right]^{*}}\end{array}$ & {$\left[{ }^{\circ}\right]$} & $\begin{array}{c}\text { SG } \\
{[\mathrm{mm}]}\end{array}$ & $\begin{array}{c}\text { GD } \\
{[\mathrm{mm}]}\end{array}$ & $\begin{array}{c}\text { Slope } \\
\text { error } \\
\text { [arcsec } \\
\mathrm{rms}]^{*}\end{array}$ \\
\hline $1 / 2400$ & 58542 & 3.15 & 1 & 564 & 572 & 2.2 \\
\hline
\end{tabular}

Table I: Main parameters of the grating. Values labeled with a * were measured as described in the text. $\alpha$ denotes incident angle and SG and GD the source grating distance and grating detector distance, respectively.

The slightly bigger radius of curvature compared to the initial design parameter, $57680 \mathrm{~mm}$, leads to a slightly larger value for the detector distance of $8 \mathrm{~mm}$.

The main contributions to the energy resolution $(\Delta E)$ of the spectrometer are the source size, the detector pixel size, and the RMS slope errors of the grating. Fig. 2 shows the resolving power $\left(\mathrm{E} / \Delta \mathrm{E}_{\text {eff }}\right)$ of the present design assuming a source size of $20 \mu \mathrm{m}$, a pixel size of $26 \mu \mathrm{m}$, and two RMS slope errors, the one on the present grating, 2.2 arcsec (red trace) and an achievable slope error of $0.2 \operatorname{arcsec}^{34}$ (blue trace). The improvement in resolving power due to a $5 \mu \mathrm{m}$ pixel size and a slope error of 2.2 arsec is shown by the black trace illustrating the importance of a smaller pixel detector.

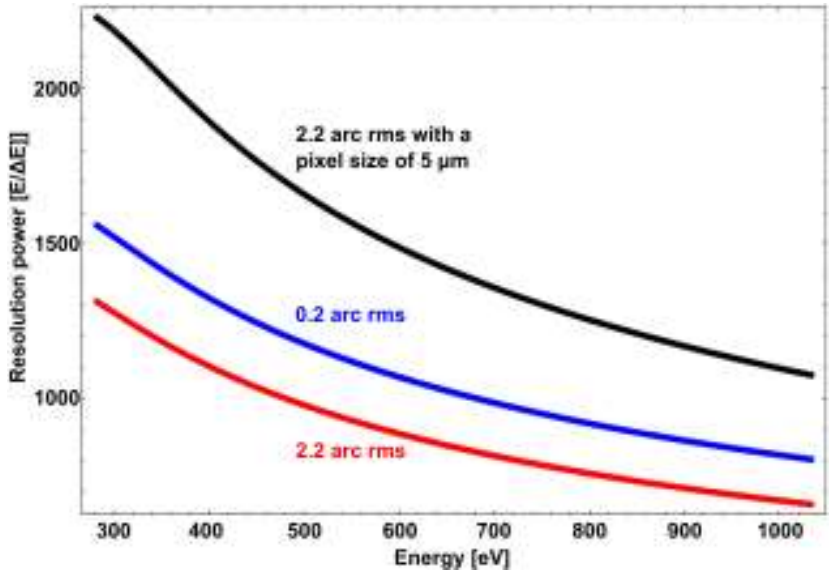

Figure 2: Resolving power assuming a $20 \mu \mathrm{m}$ source size. Red line: Detector pixel size of $26 \mu \mathrm{m}$ and RMS slope error of 2.2 arcsec; Blue line: Detector pixel size of $26 \mu \mathrm{m}$ and RMS slope error of 0.2 arcsec; Black line: Detector pixel size of $5 \mu \mathrm{m}$ pixel size detector and a RMS slope error of 2.2 arcsec. 
The manufacturer of the CCD specified the pixel size of the detector as $13 \times 13 \mu^{2}$. However, due to charge spreading the effective pixel size is around $26 \mu \mathrm{m}^{2} .^{22}$ There is the option to install the CCD at an angle of $70^{\circ}$ with respect to the focal plane to have an effective pixel size three times smaller. This will, however, reduce the photon detection efficiency. Our calculations show a possible increase in the resolving power to around 1500 with a tilted CCD at $70^{\circ}$. The development of $2 \mathrm{D}$ detectors with effective smaller pixel sizes will allow obtaining even higher resolving power with the present instrument (see Fig. 2) Nevertheless, we should point out that the resolution already achieved with this compact instrument is more than sufficient to perform RIXS studies. Higher resolution is needed to allow resolving e.g. phonon modes, yet it requires a much larger instrument.

Ray-tracing calculations were performed to determine the tolerances on the angle of incidence on the grating and the distance from the source to the grating using the VLS parameters given by the manufacturer. These results are summarized in figure 3 .

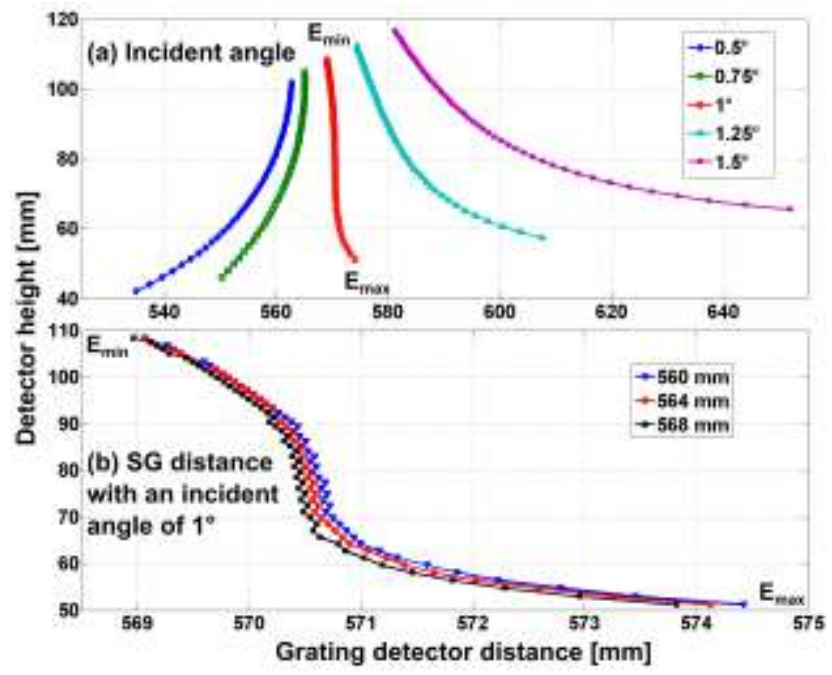

Figure 3: a) Focal curves for different angles of incidence and $S G=564 \mathrm{~mm}$. b) Focal curves for different SG distances and $1^{\circ}$ angle of incidence.

Figure 3 shows the focal plane simulation for different incident angles (a) and the focal plane for $1^{\circ}$ incident angle with different SG distances (b).

From Figure 3 it is obvious that changes in the incident angle of $\pm 0.25^{\circ}$ will severely deviate from the ideal focal curve (almost linear) whereas changes of a $\pm 4 \mathrm{~mm}$ in the SG distance are not significant.

Grating efficiency calculation performed utilizing REFLEC ${ }^{36}$ showed efficiency around $4.8 \%$ at $550 \mathrm{eV}$. Yet, due to the groove profile the actual efficiency can be lower.

\section{INSTRUMENT DESIGN}

The design of the spectrometer uses the optical lay out given by the grating manufacturer for the spherical varied line spacing (SVLS) grating HITACHI (001-0659) but with a slightly larger value for the detector distance, $8 \mathrm{~mm}$, based on the results presented in section II.

The detection of soft X-rays and the need for good resolution presents several experimental challenges. Besides the vacuum environment, the optical set up is very crucial to vibrations, the source position, the geometry of the grating with respect to the entrance slit, and the CCD photon detection system. The forces due to vacuum have to be taken into account in the overall instrument design. Therefore, to ensure high stability of the optics and the resolution of the spectrometer, the basic design uses a three bar system (see Figure 4).
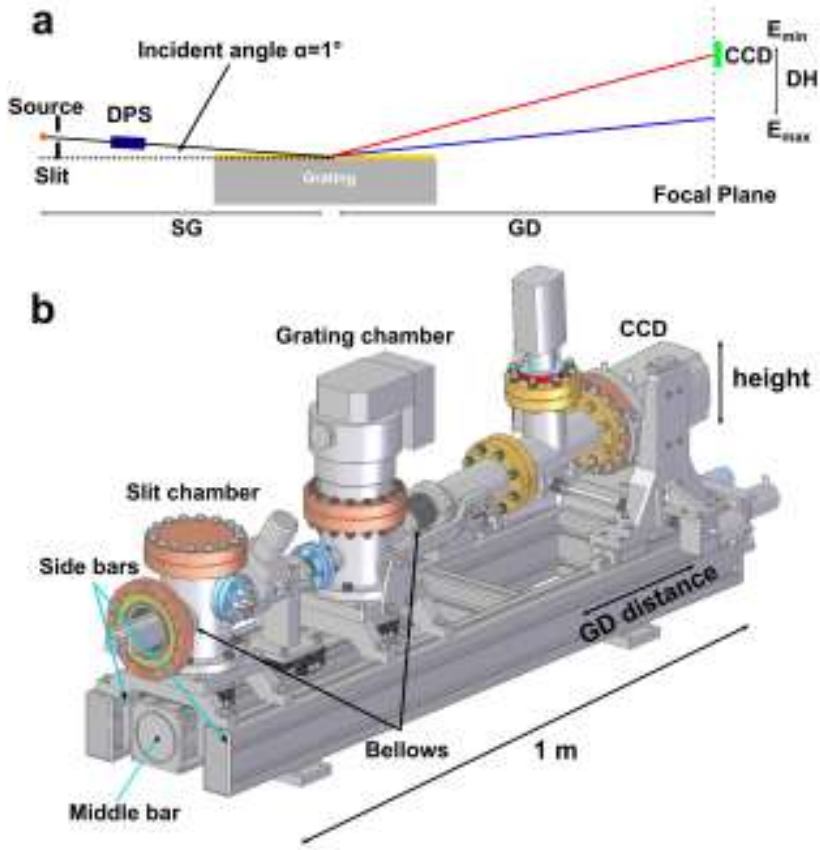

Figure 4: Schematic drawing of the alignment (a) and the spectrometer showing the mechanical structure (b). The spectrometer has a total length of around one meter, which is very compact and easy to adjust to various experimental chambers with a CF 100 port.

The side bars carry the weight of the spectrometer vacuum housing and compensate the vacuum forces. The slit system and grating holder are connected to the middle bar. There are two chambers: one contains the slit (slit chamber) and the other the grating (grating chamber). Inside the chambers, bellows minimize the vibrations of the optical elements. The detector has not been placed on the middle bar because from the design the vacuum forces can only act in vertical direction, which is compensated by a changeable detector height. Bellows in the design provide variable source - grating distance (SG), grating detector distance (GD) and detector height (DH).

A high accurate guidance for the DH and GD distances ensures high reproducibility. The slit-grating distance has 
been fixed to $564 \mathrm{~mm}$, the value specified for this grating by the manufacturer This also fixes the height difference between the grating and the slit defined by the $1^{\circ}$ degree angle of incidence on the grating. We measured the height difference and calculated an incident angle of $1.05^{\circ}$, which varies $0.05^{\circ}$ from the recommended value but the difference is negligible.

The vertical acceptance of the grating is approximately $0.11^{\circ}$ and the horizontal is around $2.89^{\circ}$.

The source (sample) - slit distance is minimized to a typical value below $1 \mathrm{~mm}$, which according to the simulations shown in figure 3 has a negligible effect on the focal curve. To record a spectrum around a given photon energy, the detector is positioned at the optimum GD distance and appropriate height (see Fig. 4a). Both motions are motor controlled with a resolution better than $10 \mu \mathrm{m}$. This design ensures high reproducibility and stability of the detector's position. The spectrometer operation in the energy range $210 \mathrm{eV}-1250 \mathrm{eV}$ in first order requires a GD variation between $562 \mathrm{~mm}$ and $582 \mathrm{~mm}$ and a height change of $\pm 35 \mathrm{~mm}$. The energy range on the detector is around $200 \mathrm{eV}$ around the central energy. A detector with a bigger chip size would lead to an increase in the energy range.

The turbo molecular pump installed on top of the grating chamber keeps the spectrometer pressure below $10^{-7}$ mbar. A differential pumping stage (DPS) is installed between the slit and the grating. Preliminary tests have shown that with the DPS one can keep three orders of magnitude lower pressure in the CCD chamber than in the sample side. This will allow performing experiments on gas and liquid jet samples, where the vacuum conditions hardly get better than $10^{-4}$ mbar in the interaction point, without inserting partially transmission foils to isolate the vacuum in the CCD. A higher pressure difference is achievable with a second turbo molecular pump connected to the slit chamber. An additional advantage of the mechanical design besides minimizing the vibration, high stability, and translation reproducibility is the simple exchange of the VLS grating.

\section{PERFORMANCE}

The test experiments have been performed at the soft $\mathrm{X}$-ray beamline P04 at PETRA III. ${ }^{37}$ The spectrometer is installed perpendicular to the incoming circular polarized synchrotron beam and in the horizontal plane. For energy calibration and determination of the resolving power we used the so-called elastic peak, which is basically the elastic scattering of the incoming photon on $\mathrm{SiO}_{2}$ and $\mathrm{MgCl}_{2}$ hexahydrate. The advantage of using the elastic scattering signal is that the energy resolution of the incoming beam is determined by the beamline characteristics, but at the cost of low cross section. The beamline slit was set at $1000 \mu \mathrm{m}$, which corresponds to an energy resolution $\Delta \mathrm{E}_{\text {beaml }}$ of around $0.55 \mathrm{eV}$ at $550 \mathrm{eV}$.
The effective resolution of the spectrometer, $\Delta \mathrm{E}_{\mathrm{spec}}$, was obtained from:

$$
\frac{\Delta E_{\text {spec }}}{E}=\sqrt{\left(\frac{\Delta E_{\text {total }}}{E}\right)^{2}-\left(\frac{\Delta E_{\text {beaml }}}{E}\right)^{2}}
$$

The measured total energy resolution is $0.77 \mathrm{eV}$ at $550 \mathrm{eV}$, which leads to an effective spectrometer energy resolution of $0.54 \mathrm{eV}$ corresponding to a resolving power of $\sim 1020$ (see Figure 5).

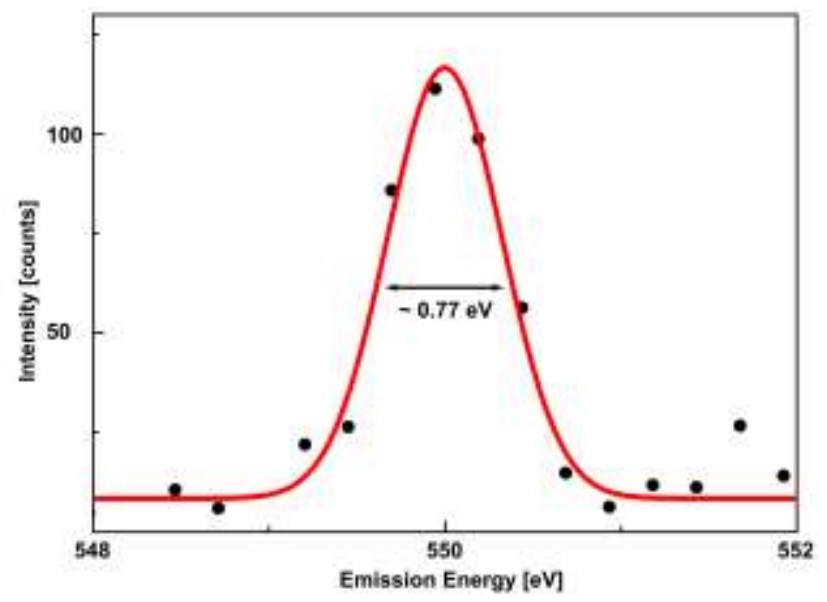

Figure 5: The Gaussian fit (red) of the elastic peak (dots) of $\mathrm{MgCl}_{2}$ hexahydrate shows a total energy resolution of around $0.77 \mathrm{eV}$ at $550 \mathrm{eV}$ incident energy. The total data acquisition time was $80 \mathrm{~min}$.

The data acquisition time was $80 \mathrm{~min}$ with the beamline delivering $3 \times 10^{13} \mathrm{ph} / \mathrm{sec}$ onto a spot size of $0.350 \mathrm{~mm} \times 7$ $\mathrm{mm}$ and a spectrometer slit at around $20 \mu \mathrm{m}$. The large mismatch between the slit size and the beamline spot size is one of the reasons causing the long data acquisition time. Figure 6 illustrates the elastic peak for an incoming energy of $540 \mathrm{eV}$.

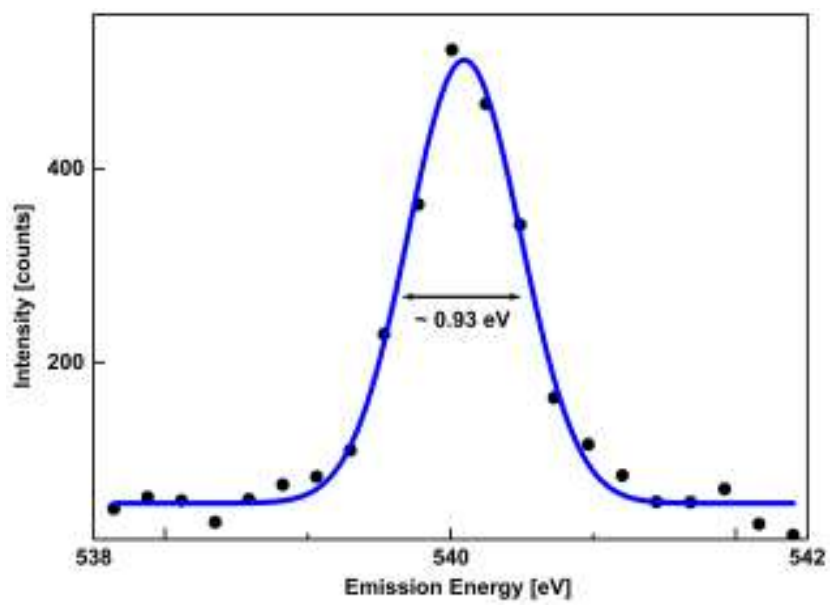

Figure 6: The Gaussian fit (blue) of the elastic peak (dots) shows a total energy resolution of around $0.93 \mathrm{eV}$ at 540 $\mathrm{eV}$ incident energy. The total data acquisition time is $3.5 \mathrm{~h}$. 
The overall resolution is $0.93 \mathrm{eV}$. The lower resolving power is mainly due to a bigger beamline slit size, which leads to an increased signal at the expense of energy resolution. At the P04 beamline of PETRA III the beam spot can now be focused down to $10 \times 10 \mu \mathrm{m}^{2}$ giving the advantage to operate the spectrometer with a fully open entrance slit in a "slitless" mode to enhance the detection efficiency without degrading the resolution.

\section{CONCLUSION}

We presented a newly designed compact grating spectrometer using a spherical VLS grating, which covers the energy range of light elements, like carbon, nitrogen and oxygen $\mathrm{K}-\alpha_{1,2}$ and the $\mathrm{L}-\alpha_{1,2}$ of $3 \mathrm{~d}$ transitions metals. The spectrometer can achieve a resolving power of around 1000 , but it has the potential to enhance the resolving power by $50 \%$. The spectrometer is optimal for RIXS studies especially in systems with high vapor pressure, i.e. systems in liquid phases. Its compact design allows utilizing this spectrometer at different experimental setups.

\section{ACKNOWLEDGEMENTS}

The authors gratefully acknowledge the financial support from SFB755 "Nanoscale Photonic Imaging" and SFB 1073 "Atomic Scale Control of Energy Conversion" of the German Science Foundation (DFG), the Max Planck Institute of Biophysical Chemistry, Deutsches ElektronenSynchrotron and Helmholtz Zentrum Berlin. S.T. is grateful to the Funds of the Chemical Industry. We thank the staff of P04 and S. Klumpp for their continuous support and T. Baumann and J. R. Crespo López-Urrutia for valuable discussions.

\section{CORRESPONDING AUTHORS}

\section{a) zhong.yin@desy.de \\ b) simone.techert@desy.de}

\section{REFERENCES}

${ }^{1}$ L. Ament, M. van Veenendaal, T. Devereaux, J. Hill, and J. van den Brink, Rev. Mod. Phys. 83, 705 (2011).

${ }^{2}$ A. Kotani and S. Shin, Rev. Mod. Phys. 73, 203 (2001).

${ }^{3}$ A. Föhlisch, P. Feulner, F. Hennies, A. Fink, D. Menzel, D. SanchezPortal, P.M. Echenique, and W. Wurth, Nature 436, 373 (2005).

${ }^{4}$ F. Gel'mukhanov and A. Hans, Phys. Rep. 312, 87 (1999).

${ }^{5}$ Z. Yin, I. Rajkovic, K. Kubicek, W. Quevedo, A. Pietzsch, P. Wernet, A. Föhlisch, and S. Techert, J. Phys. Chem. B 118, 9398 (2014).

${ }^{6}$ S. Schreck, M. Beye, J. a. Sellberg, T. McQueen, H. Laksmono, B.

Kennedy, S. Eckert, D. Schlesinger, D. Nordlund, H. Ogasawara, R.G. Sierra, V.H. Segtnan, K. Kubicek, W.F. Schlotter, G.L. Dakovski, S.P. Moeller, U. Bergmann, S. Techert, L.G.M. Pettersson, P. Wernet, M.J. Bogan, Y. Harada, A. Nilsson, and A. Föhlisch, Phys. Rev. Lett. 113, 153002 (2014).

${ }^{7}$ K. Kunnus, I. Josefsson, S. Schreck, W. Quevedo, P. S. Miedema, S. Techert, F. M. F. De Groot, M. Odelius, P. Wernet, and A. Föhlisch, J. Phys. Chem. B 117, 16512 (2013).

${ }^{8}$ T. Tokushima, Y. Horikawa, H. Arai, Y. Harada, O. Takahashi, L.G.M. Pettersson, A. Nilsson, and S. Shin, J. Chem. Phys. 136, 0445171 (2012).
${ }^{9}$ L. Weinhardt, M. Blum, O. Fuchs, a. Benkert, F. Meyer, M. Bär, J.D. Denlinger, W. Yang, F. Reinert, and C. Heske, J. Electron Spectros. Relat. Phenomena 188, 111 (2013).

${ }^{10}$ J.-E. Rubensson, F. Hennies, and A. Pietzsch, J. Electron Spectros. Relat. Phenomena 188, 79 (2013).

${ }^{11}$ M. Beye, S. Schreck, F. Sorgenfrei, C. Trabant, N. Pontius, C. Schüßler-Langeheine, W. Wurth, and A. Föhlisch, Nature 501, 191 (2013).

${ }^{12}$ E.F. Aziz, J. Electron Spectros. Relat. Phenomena 177, 168 (2010).

${ }^{13}$ R. Mitzner, J. Rehanek, J. Kern, S. Gul, J. Hattne, T. Taguchi, R. Alonso-mori, R. Tran, C. Weniger, H.S. Der, W. Quevedo, H Laksmono, R.G. Sierra, G. Han, B. Lassalle-kaiser, S. Koroidov, K. Kubicek, S. Schreck, K. Kunnus, M. Brzhezinskaya, A. Firsov, M.P. Minitti, J.J. Turner, S. Moeller, N.K. Sauter, M.J. Bogan, D. Nordlund, W.F. Schlotter, J. Messinger, A. Borovik, S. Techert, F.M.F. De Groot, A. Föhlisch, A. Erko, U. Bergmann, V.K. Yachandra, P. Wernet, and J. Yano, J. Phys. Chem. Lett. 4, 3641 (2013).

${ }^{14}$ P. Wernet, K. Kunnus, I. Josefsson, I. Rajkovic, W. Quevedo, M. Beye, S. Schreck, S. Grübel, M. Scholz, D. Nordlund, W. Zhang, R.W.

Hartsock, W.F. Schlotter, J.J. Turner, B. Kennedy, F. Hennies, F.M.F. de Groot, K.J. Gaffney, S. Techert, M. Odelius, and A. Föhlisch, Nature 520, 78 (2015).

${ }^{15}$ P. Glatzel and U. Bergmann, Coord. Chem. Rev. 249, 65 (2005).

${ }^{16}$ H.H. Johann, Zeitschrift Für Phys. 69, 185 (1931).

${ }^{17}$ L. v. Hámos, Naturwissenschaften 20, 705 (1932).

${ }^{18}$ T. A. Callcott, K.L. Tsang, C.H. Zhang, D.L. Ederer, and E.T. Arakawa, Rev. Sci. Instrum. 57, 2680 (1986).

${ }^{19}$ J. Nordgren, G. Bray, S. Cramm, R. Nyholm, J.-E. Rubensson, and N. Wassdahl, Rev. Sci. Instrum. 60, 1690 (1989).

${ }^{20}$ C. Dallera, E. Puppin, G. Trezzi, N. Incorvaia, a Fasana, L. Braicovich, N. B. Brookes, and J. B. Goedkoop, J. Synchrotron Radiat. 3, 231 (1996).

${ }^{21}$ S.G. Chiuzbăian, C.F. Hague, A. Avila, R. Delaunay, N. Jaouen, M. Sacchi, F. Polack, M. Thomasset, B. Lagarde, A. Nicolaou, S. Brignolo, C. Baumier, J. Lüning, and J.-M. Mariot, Rev. Sci. Instrum. 85, 043108 (2014)

${ }^{22}$ G. Ghiringhelli, A. Piazzalunga, C. Dallera, G. Trezzi, L. Braicovich, T. Schmitt, V.N. Strocov, R. Betemps, L. Patthey, X. Wang, and M. Grioni, Rev. Sci. Instrum. 77, 113108 (2006).

${ }^{23}$ Y. Harada, M. Kobayashi, H. Niwa, Y. Senba, H. Ohashi, T. Tokushima, Y. Horikawa, S. Shin, and M. Oshima, Rev. Sci. Instrum. 83, 013116 (2012)

${ }^{24}$ Y.-D. Chuang, J. Pepper, W. McKinney, Z. Hussain, E. Gullikson, P. Batson, D. Qian, and M.Z. Hasan, J. Phys. Chem. Solids 66, 2173 (2005).

${ }^{25}$ O. Fuchs, L. Weinhardt, M. Blum, M. Weigand, E. Umbach, M. Bär, C. Heske, J. Denlinger, Y.-D. Chuang, W. McKinney, Z. Hussain, E. Gullikson, M. Jones, P. Batson, B. Nelles, and R. Follath, Rev. Sci. Instrum. 80, 063103 (2009).

${ }^{26}$ F. Hennies, A. Pietzsch, M. Berglund, A. Föhlisch, T. Schmitt, V. Strocov, H.O. Karlsson, J. Andersson, and J.-E. Rubensson, Phys. Rev. Lett. 104, 1 (2010).

${ }^{27}$ M. E. Dinardo, a. Piazzalunga, L. Braicovich, V. Bisogni, C. Dallera, K. Giarda, M. Marcon, a. Tagliaferri, and G. Ghiringhelli, Nucl. Instruments Methods Phys. Res. Sect. A Accel. Spectrometers, Detect. Assoc. Equip. 570, 176 (2007)

${ }^{28}$ H. Noda, T. Namioka, and M. Seya, J. Opt. Soc. Am. 64, 1031 (1974).

${ }^{29} \mathrm{http} / / / \mathrm{www}$.andor.com/scientific-cameras/ikon-ccd-camera-series

${ }^{30}$ T. Harada, K. Takahashi, H. Sakuma, and A. Osyczka, Appl. Opt. 38, 2743 (1999)

${ }^{31}$ G. Binnig, CF. Quate, C. Gerber, Phys. Rev. Lett. 56, 930 (1986)

${ }^{32}$ E. Meyer, Progress in Surface Science, 41, 3 (1992)

${ }^{33}$ J. Wernecke, H. Okuda, H. Ogawa, F. Siewert, M. Krumrey, Macromolecules, 47, 5719 2014)

${ }^{34}$ F. Siewert, J. Buchheim, T. Zeschke, G. Falkenberg, R. Sankari, J Synchrotron Rad. 21, 968 (2014).

${ }^{35}$ F. Siewert, Journal of Physics: Conf. Series, 425, 152001 (2013).

${ }^{36} \mathrm{~F}$. Schäfers andM.Krumrey, "REFLEC-A program to calculate VUV and soft $\mathrm{x}$-ray optical elements and synchrotron radiation beamlines," Technical Report 201 (BESSY, 1996), pp. 1-17.

${ }^{37}$ J. Viefhaus, F. Scholz, S. Deinert, L. Glaser, M. Ilchen, J. Seltmann, P. Walter, and F. Siewert, Nucl. Instr. Methods Phys. Res. A. 710, 151 (2013). 